\title{
Surface states and arcless angles in twisted Weyl semimetals
}

\author{
Ganpathy Murthy, ${ }^{1}$ H. A. Fertig, ${ }^{2}$ and Efrat Shimshoni ${ }^{3}$ \\ ${ }^{1}$ Department of Physics and Astronomy, University of Kentucky, Lexington, Kentucky 40506-0055, USA \\ ${ }^{2}$ Department of Physics, Indiana University, Bloomington, Indiana 47405, USA \\ ${ }^{3}$ Department of Physics, Bar-Ilan University, Ramat-Gan 52900, Israel
}

(Received 26 September 2019; accepted 28 January 2020; published 26 March 2020)

\begin{abstract}
Fermi arc states are features of Weyl semimetal (WSM) surfaces which are robust due to the topological character of the bulk band structure. We demonstrate that Fermi arcs may undergo profound restructurings when surfaces of different systems with a well-defined twist angle are tunnel coupled. The twisted WSM interface supports a moiré pattern which may be approximated as a periodic system with a large real-space unit cell. States bound to the interface emerge, with interesting consequences for the magneto-oscillations expected when a magnetic field is applied perpendicular to the system surfaces. As the twist angle passes through special "arcless angles," for which open Fermi arc states are absent at the interface, Fermi loops of states with no connection to bulk states appear. Such states have interesting resonance signatures in the optical conductivity of the system in a magnetic field perpendicular to the interface. These signatures are independent of the bulk thickness or disorder.
\end{abstract}

DOI: 10.1103/PhysRevResearch.2.013367

\section{INTRODUCTION}

Weyl semimetals (WSM's) are three-dimensional materials which host electronic structures with unusual, robust topological properties. Their bulk band structures contain an even number of "Weyl points," locations where the constant energy surfaces shrink to a point, which act as sources or sinks of Berry's flux through two-dimensional surfaces surrounding them [1,2]. Their presence in such materials leads to the nonconservation of currents associated with individual Weyl nodes in collinear electric and magnetic fields, and an associated negative longitudinal magnetoresistance [1,3]. WSM's can arise in spin-orbit coupled systems with either broken inversion symmetry [4] or time-reversal symmetry [5-8]. Inversion-broken WSM's were discovered in 2015 [9-12], while the time-reversal broken WSM's have been found very recently [13-16]. For the latter, anomalous Hall effects $[17,18]$ are expected, as well as nonlocal transport properties [19-21]. Indeed, these systems yield relatively clean surfaces upon cleaving [13-16], allowing clear signatures of the surface states supporting such phenomena to be identified.

Surfaces of WSM's have topologically protected "Fermi arc" states [5,22]. At zero energy (defined as the energy of the Weyl points), Fermi arcs in the surface Brillouin zone (BZ) are one or more curves that connect the projections of the Weyl points onto the surface BZ, with the energy dispersing perpendicular to the arc. At the arc end points, the penetration

Published by the American Physical Society under the terms of the Creative Commons Attribution 4.0 International license. Further distribution of this work must maintain attribution to the author(s) and the published article's title, journal citation, and DOI. depth of the surface state diverges, allowing for exchange between surface and bulk currents. In a slab placed in a magnetic field perpendicular to the surfaces, current flows in opposite directions along Fermi arcs on each surface, connected by chiral states through the bulk, to form closed loops [23]. These periodic orbits are expected to generate magneto-oscillations in measurable properties of a slab of a WSM [24,25]. For a thin enough slab, the Fermi arcs on opposite surfaces may tunnel-couple, and generate a quantized Hall effect [26]. Notably, because these effects involve a bulk component, they are sensitive to bulk thickness or disorder [19,20,25].

In the present study we propose a tunable reconstruction that generates states entirely confined to the surface, with their experimental signatures being insensitive to the bulk thickness or disorder. Because of their topological protection, scattering among surface states that can degrade their behaviors should be suppressed, leading to a relative robustness of phenomena associated with these states. Specifically, we consider the fate of Fermi arc states when surfaces from two separate WSM's are twisted with respect to one another, and then tunnel coupled. We demonstrate that a rich set of possible Fermi arc reconstructions emerges in such systems, due to the formation of moiré patterns which can be approximated by two-dimensional lattices. The effects of moiré patterns in twisted bilayer graphene [27,28] and other two-dimensional van der Waals bonded systems [29,30] have recently been demonstrated to yield remarkable physical effects [31,32], albeit of a form very different from what we discuss below. While tunnel-coupled Weyl semimetals have been considered previously $[33,34]$, their focus on highly aligned crystal structures misses the remarkable moiré physics such systems may host.

In the settings we consider, tunneling between states on the two WSM surfaces can occur directly between states of the same crystal momentum ("direct tunneling") or between 


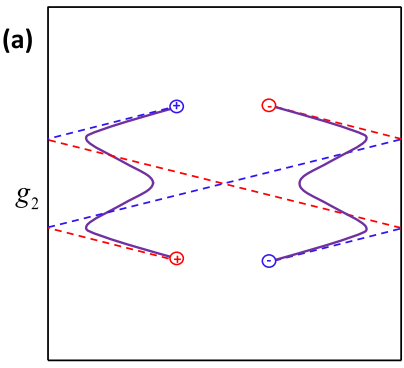

$g_{1}$
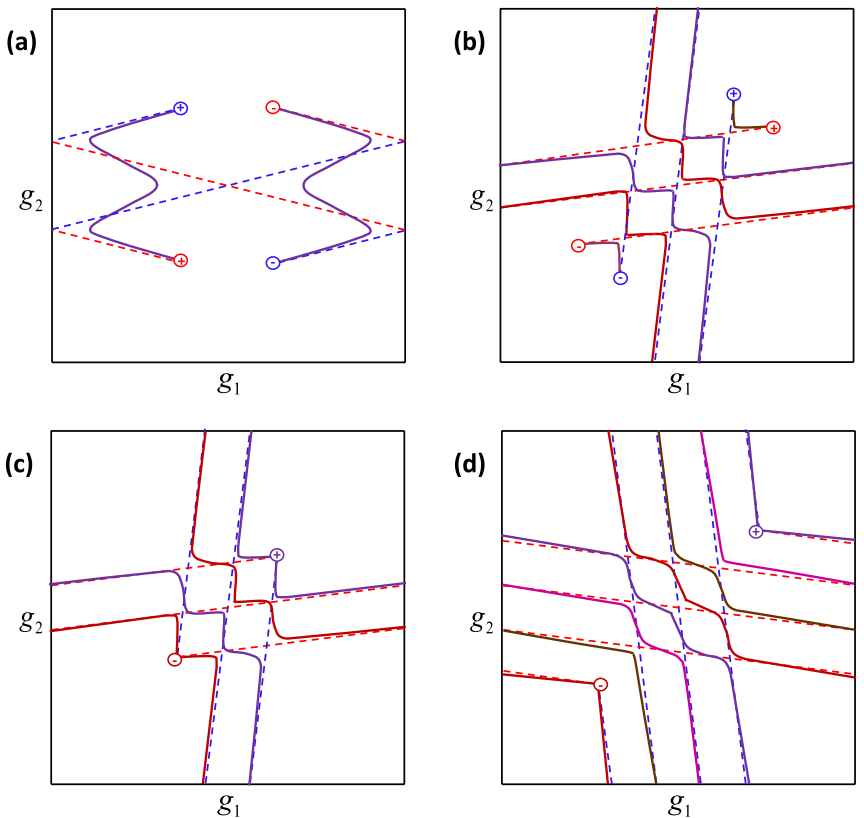

FIG. 1. Reconstructed Fermi arcs patterns in the moiré Brillouin zone for various values of the twist angle $\theta$ : (a) $\theta<\pi / 4$; (b) an arbitrary $\pi / 4<\theta<\pi / 2$; (c) the arcless angle $\theta_{1}$, where two closed loops are generated; (d) the arcless angle $\theta_{-2}$, yielding two distinct pairs of closed loops (of which only one pair connects to the Weyl nodes). Dashed blue (red) lines represent the original arcs on the top (bottom) WSM slab.

states whose wave vectors differ by linear combinations of the reciprocal lattice vectors in each layer ("umklapp tunneling"). As long as the range of the interlayer tunneling is not too short, an effective periodic problem emerges, defined by a moiré Brillouin zone spanned by the two shortest scattering vectors. We find that, generically, the coupled Fermi arcs within a moiré Brillouin zone will oscillate in direction [as shown in Fig. 1(a)] and typically connect Weyl nodes of the two different slabs [33,34]. For some ranges of angles, one finds that Fermi arcs reconstruct to form open arcs connecting the Weyl nodes, coexisting with arcs that close upon themselves. For special "arcless angles," the interface hosts only closed Fermi loops. For relatively weak interlayer tunnel coupling, some of these loops include points at the surface projections of the Weyl points, while for stronger tunnel coupling all the Fermi loops at the interface are completely disconnected from the Weyl points. A summary of these different possibilities is illustrated in Fig. 1.

The existence of closed Fermi loops at the arcless angles is particularly interesting. For sufficiently clean systems this means the interface hosts two types of conducting channels. Type (i) channels go through the Weyl point projections and have significant overlap with the bulk at the Weyl points. In contrast, type (ii) channels never intersect the Weyl point projections. Hence they are confined to the interface and are insensitive to bulk thickness or disorder. In the presence of a perpendicular magnetic field, currents associated with type (ii) channels should flow along closed loops in the surface BZ. Although they present open orbits in real space-and are thus not expected to induce density-of-states oscillations-they involve periodic motion due to the meandering of the orbits through the moiré Brillouin zone, with periods inversely proportional to the magnetic field. This implies that the interface will present resonances in its optical conductivity $\sigma(\omega)$ that reflect the orbit periods, as occurs in quasi-one-dimensional materials [35]. Type (i) orbits similarly should present oscillations in $\sigma(\omega)$; however, because they are closed in real space they should additionally induce density-of-states magnetooscillations which can be detected in thermodynamic quantities at zero frequency [23]. For type (i) orbits the resonance frequency in $\sigma(\omega)$ is expected to jump as the twist angle crosses an arcless angle. For type (ii) orbits, the frequency itself evolves continuously as a function of twist angle, but the amplitude of $\sigma(\omega)$ should jump as arcless angles are crossed and new closed Fermi loops are created.

We now turn to a more detailed discussion of our results.

\section{MODEL HAMILTONIAN AND SURFACE STATES}

Our starting point is a simple two-band model [6] (with broken time-reversal symmetry) of a WSM hosting two Weyl nodes, supporting a single Fermi arc on any surface for which the projections of the Weyl points do not overlap in the surface Brillouin zone. The underlying crystal is cubic (with lattice constant as our length unit), and the bulk Hamiltonian as a function of crystal momentum takes the form

$$
H_{0}\left(\mathbf{k}, k_{z}\right)=2 \sum_{\mu=x, y, z} f_{\mu} \sigma_{\mu} .
$$

In this expression, $\mathbf{k}$ is a two-dimensional momentum, $\sigma_{\mu}$ are Pauli matrices, and $f_{x}=t\left(2+\cos k_{0}-\cos k_{x}-\cos k_{y}-\right.$ $\left.\cos k_{z}\right) \equiv t\left(1-\cos k_{z}\right)+\tilde{f}_{x}, f_{y}=t \sin k_{y}$, and $f_{z}=t^{\prime} \sin k_{z}$. The bulk Weyl points are $\left( \pm k_{0}, 0,0\right)$. To simplify our notation, we hereon set $t=1$. The existence and form of surface states may be found by going to the continuum limit in one direction, which we take here to be $\hat{z}$. Expanding $H_{0}\left(\mathbf{k}, k_{z}\right)$ to second order in $k_{z}$, we can look for evanescent solutions by taking $k_{z} \rightarrow i \lambda$. Within this procedure [36-38], four values of $\lambda$ satisfying $H_{0}(\mathbf{k}, i \lambda) \boldsymbol{\Phi}=E \boldsymbol{\Phi}$ may be found, two of which produce wave functions that vanish as $z \rightarrow \infty$. One must then choose the value of $E$ such that the eigenvector $\boldsymbol{\Phi}$ is the same for both these values of $\lambda$, so that a linear combination may be formed satisfying vanishing boundary conditions at $z=0$. (Details are provided in Appendix A.) Three points resulting from this analysis are particularly relevant to what follows: (i) For the model defined by Eq. (1), the procedure described above can only be carried through for $\left|k_{x}\right|<k_{0}$. Thus the surface states are present on an open arc in the surface momentum space. (ii) Eigenstates of $H_{0}$ of this form satisfy $E=f_{y}\left(k_{y}\right) \approx k_{y}$, so that the states have a velocity along the $\hat{y}$ direction. (iii) The eigenvectors that result from the analysis are spin polarized along the $\sigma_{y}$ direction. For this simple model we can also obtain surface states directly within the tight-binding model (Appendix B), yielding results consistent with the continuum approximation. The lattice analysis has strengths complementary to the continuum approach, in particular allowing the possibility of making the tunnel coupling nonperturbative, which we explore in detail in Appendices $\mathrm{C}$ and D. 


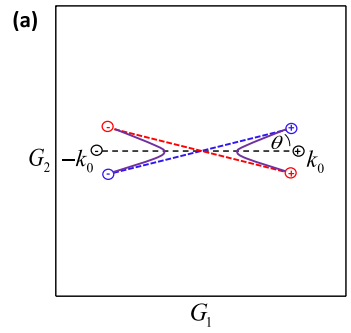

(b)

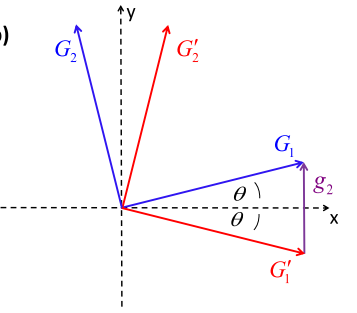

(c)

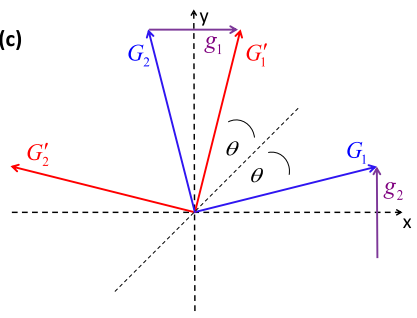

FIG. 2. (a) Zero-energy contours (solid purple curves) resulting from the reconstruction of Fermi arcs (dashed lines) on the coupled surfaces of twisted WSM's. (b) Primitive lattice wave vectors of the top and bottom surfaces for a small twist angle $(\theta<\pi / 4)$. (c) Primitive lattice vectors of the top and bottom surfaces for a twist angle $\pi / 4<\theta<\pi / 2$; the wave vectors $\mathbf{g}_{1}, \mathbf{g}_{2}$ define the moiré Brillouin zone.

\section{TWISTED WSM'S AND TUNNEL-COUPLED FERMI ARCS}

Consider a pair of semi-infinite WSM's, one exposing a top surface and the other a bottom surface, each with a Fermi arc oriented at angles $\pm \theta$ with respect to the $\hat{x}$ axis. For our model, the Fermi arc state dispersions near zero energy can be written in the form $E_{T, B}(\mathbf{k}) \approx \mathbf{v}_{T, B} \cdot \mathbf{k}$, with $\mathbf{v}_{T} \cdot \mathbf{v}_{b}=-v_{0}^{2} \cos 2 \theta$. In a long-wavelength model in which the tunneling amplitude is uniform across the surfaces, the two-dimensional crystal momentum will be conserved. Projecting into the Fermi arc states of the two surfaces, the coupled Hamiltonian may be modeled as

$$
H_{c}=\left(\begin{array}{cc}
\mathbf{v}_{T} \cdot \mathbf{k} & w_{d} \\
w_{d} & \mathbf{v}_{B} \cdot \mathbf{k}
\end{array}\right),
$$

where we have assumed $w_{d}$ is real. In principle, after projection onto the surface states $w_{d}$ is a function of $\mathbf{k}$, and should vanish at the positions of the arc end points, for which one of the inverse length scales $\lambda$ in the surface state construction vanishes, indicating the Fermi arc state crosses over into a bulk state. However, $w$ is most important near $k=$ 0 where the Fermi arcs cross, and is nonvanishing at that location. Diagonalizing $H_{c}$ yields energies $E_{ \pm}=-v_{0} \sin \theta k_{x} \pm$ $\left[v_{0}^{2} \cos ^{2} \theta k_{y}^{2}+w_{d}^{2}\right]^{1 / 2}$, whose zero-energy contours are illustrated in Fig. 2(a). The reconstructed Fermi arcs now connect Weyl nodes on opposite sides of $z=0$.

In reality, the two surfaces each have a square lattice structure, and this makes the physics notably richer. Primitive lattice vectors for the top (bottom) surface may be written as $\mathbf{G}_{1}^{(')}$ and $\mathbf{G}_{2}^{\left({ }^{\prime}\right)}$ as illustrated in Fig. 2(b) for a small twist angle. A simple model incorporating this [27] assumes that the tunneling amplitude between atoms in different systems depends only on the total distance between them; summing over the two-dimensional direct lattice vectors then leads to tunneling processes in which the in-plane wave vector scatters by a difference in reciprocal lattice vectors, $\mathbf{g}_{i j} \equiv$ $n_{i} \mathbf{G}_{i}-n_{j}^{\prime} \mathbf{G}_{j}^{\prime}$, with $i, j=1,2$ and $n_{i}, n_{j}^{\prime}$ integers. The largest amplitudes for such umklapp tunneling processes occur when one of these integers vanishes and the other is \pm 1 . These processes may be regarded as renormalizations of the direct tunneling process. Qualitatively new behavior emerges when $\left|n_{i}\right|=\left|n_{j}^{\prime}\right|=1$, and translation of the Fermi arcs by $\mathbf{g}_{i j}$ causes them to overlap at new locations in momentum.

The Hamiltonian for states projected onto the Fermi arcs in this case takes the form

$$
H_{c}=\left(\begin{array}{cc}
\mathbf{v}_{T} \cdot\left(\mathbf{k}+\mathbf{g}_{i j}\right) & w_{u} \\
w_{u} & \mathbf{v}_{B} \cdot \mathbf{k}
\end{array}\right),
$$

where $w_{u}$ is a tunneling amplitude associated with the umklapp process defined by $\mathbf{g}_{i j}$. Within the assumptions discussed above we expect $\left|w_{u}\right|<\left|w_{d}\right|$; however, for values of $\mathbf{k}$ such that $\mathbf{v}_{T} \cdot\left(\mathbf{k}+\mathbf{g}_{i j}\right) \approx \mathbf{v}_{B} \cdot \mathbf{k}$ its effect cannot be neglected. By retaining just the vectors $\mathbf{g}_{i j}$ that generate such degeneracies, one can describe the system in terms of an effective superlattice, which may be viewed as an approximation to the moiré pattern [27] expected to appear at the interface between the two WSM's. Using this paradigm, we next turn to some of the interesting reconstructions of the Fermi arcs that result from this physics.

\section{RESULTS}

We begin by considering the situation for small $\theta$. In this case there is a single pair of collinear scattering vectors, $\pm \mathbf{g}_{1}$, where $\left|\mathbf{g}_{1}\right|=2 \sin \theta\left|\mathbf{G}_{1}\right|$ [see Fig. 2(b)], which are relevant to the Fermi arc restructuring. In this case the system may be regarded as a one-dimensional superlattice, and an example of the Fermi arc behavior is illustrated in Fig. 1(a). We assume the "bare" Fermi arcs to be short compared to the surface Brillouin zone sizes of the individual layers. For small enough $\theta$ the reconstructed Fermi arcs oscillate back and forth repeatedly, ultimately connecting a Weyl node of the lower system to one for the upper system. As $\theta$ increases the number of oscillations decreases, and ultimately the reconstructed Fermi arcs will turn back once around $\mathbf{k}=0$ through a direct process to connect nodes in opposite layers.

An interesting consequence of this behavior is its effect on magneto-oscillations expected for WSM slabs [23]. For two rotated slabs tunnel coupled in this way, in a magnetic field $B$ perpendicular to the interface, semiclassical orbits will pass through the reconstructed arcs. While the total arc length and the effective Lorentz forces along most sections of the arcs are rather similar to their values for uncoupled arcs, at the crossing points the Lorentz force becomes $\sim B v_{0} \sin \theta$, leading to a lengthening of the total orbit period at small $\theta$, both because of the slow velocity at an orbital turning point, and because of the proliferation of the number of such turns as $\theta \rightarrow 0$. This should lead to an anomalous increase of the magneto-oscillation period at small twist angles, which is eliminated as the twist angle increases, both continuously as the velocity around the turning points increases and in steps as the "switchbacks" in the interface orbits are eliminated. 
Figure 1(b) illustrates the situation for a twist angle satisfying $2 \theta \sim \pi / 2$. The relevant scattering wave vectors in this case are $\pm \mathbf{g}_{1}$ and $\pm \mathbf{g}_{2}$, where $\mathbf{g}_{1} \equiv \mathbf{G}_{1}^{\prime}-\mathbf{G}_{2}, \mathbf{g}_{2} \equiv \mathbf{G}_{2}^{\prime}+\mathbf{G}_{1}$ [see Fig. 2(c)]. These wave vectors form an effective small Brillouin zone $\left[\mathbf{g}_{1}, \mathbf{g}_{2}\right]$ that defines the wave vector space for the periodic approximation to the moire pattern at the twist interface. Because of the two-dimensional character of this Brillouin zone, the pattern of avoided crossings breaks up the Fermi arcs into a pair of short Fermi arcs connecting Weyl points in the two different systems, and one or more longer closed loops wrapping around the torus defined by the moiré Brillouin zone. For specific twist angles, the Weyl nodes overlap perfectly, eliminating the Fermi arcs, but leave behind the closed orbits [Figs. 1(c) and 1(d)]. These "arcless angles" satisfy the equation

$$
\tan \left(\frac{\pi}{2}-2 \theta_{n}\right)=\frac{k_{0}}{k_{0}+4 \pi n},
$$

with $n$ an integer. The situation at such angles is unique, in that the twisted WSM supports $2|n|$ closed loops of surface states. In the perturbative regime, two of these closed loops go through the Weyl point projections [type (i) loops], while $2(|n|-1)$ of them are composed of purely interface states [type (ii) loops]. The number of such closed loops changes with twist angle, becoming arbitrarily large as $2 \theta \rightarrow \pi / 2$. Note that at the arcless angles, even the two type (i) Fermi loops at the interface that nominally overlap with the Weyl point projections may actually detach from them at strong enough tunnel coupling, as we demonstrate in Appendix D for a specific geometry. In such cases, all surface states form closed loops disconnected from the Weyl point projections.

Finally we note that for relative twist angles $2 \theta \sim \pi$ and $2 \theta \sim 3 \pi / 2$, one expects to encounter restructured arc states with forms qualitatively similar to those for small twist angles and for $2 \theta \sim \pi / 2$, respectively.

\section{DISCUSSION}

The closed Fermi loops that form at twist interfaces for relative angle $2 \theta \sim \pi / 2$ of type (ii), and of both types at the arcless angles, represent some of the more surprising phenomena occurring in the twisted WSM system. A direct probe of electrons in these states involves their semiclassical dynamics in a magnetic field $\mathbf{B}$ perpendicular to the interface. The relevant equations of motion are

$$
\begin{aligned}
\frac{d \mathbf{k}}{d t} & =-e \mathbf{E}-\frac{e}{c} \mathbf{v}_{\alpha} \times \mathbf{B}, \\
\mathbf{v}_{\alpha}(\mathbf{k}) & =\nabla \varepsilon_{\alpha}(\mathbf{k}) .
\end{aligned}
$$

In these equations, $\mathbf{k}$ is contained in the moiré Brillouin zone, and $\alpha$ labels different disconnected parts of the reconstructed Fermi arcs [39]. The energy function $\varepsilon_{\alpha}(\mathbf{k})$ corresponding to the closed Fermi arc is given approximately, over most of its length, by the Fermi arc energies for the uncoupled systems, appropriately translated into the moiré Brillouin zone. For trajectories near zero energy at $\mathbf{E}=0$ and for $0 \leqslant \theta \leqslant \pi / 4$, one should set $\varepsilon_{\alpha}=E_{-}$[with $E_{-}$as defined below Eq. (2)] near degeneracy points.

First we focus on type (ii) loops. The orbits of Eqs. (5) are closed orbits in the moiré Brillouin zone, but open in real space. Magneto-oscillations in the electronic density of states are not expected; however, the periodicity of the $k$-space orbit should generate excitations of finite frequency, detectable by resonances in the optical conductivity $\sigma(\omega)$ [35].

As $\theta$ (assumed near $\frac{\pi}{2}$ ) decreases the period of the type (ii) closed orbits in $\mathbf{k}$ decreases, and the frequency of the resonance in $\sigma(\omega)$ increases smoothly as a function of $\theta$. However, every time an arcless angle is crossed, the number of type (ii) closed loops changes by 2 , which should lead to a jump in the resonance amplitude in $\sigma(\omega)$. (These statements are strictly correct for weak tunnel coupling, for which all closed loops are expected to have the same period for a given $\mathbf{B}$.) Interestingly, because the orbits generating these resonances oscillate both within the plane and perpendicular to it, they should be detectable via absorption of electromagnetic waves polarized either parallel or perpendicular to the interface [40]. Importantly, since they are confined to the interface, type (ii) loops are insensitive to bulk thickness or disorder.

For generic angles, the type (i) interface orbits that connect to the bulk Weyl points should admit more general magnetooscillations [23], with period dependent on length of the Fermi arcs at the interface, as well as those at the more remote surfaces, which will also produce resonances in $\sigma(\omega)$. As the Fermi arcs abruptly change length when $\theta$ passes through an arcless angle, we expect jumps in the resonance frequency in $\sigma(\omega)$ due to type (i) orbits. For strong enough tunnel coupling between the two slabs, at the arcless angles, the Fermi arcs may break away from the projections of the Weyl points on the surface BZ and form two additional closed loops. Such loops will exhibit a quantized Hall conductance at the interface in a perpendicular magnetic field.

Finally, we briefly comment on the situation for WSM's where the Weyl nodes appear due to broken inversion symmetry, rather than broken time-reversal symmetry. A model bulk Hamiltonian of such a system, with four bands [41], has the form [42]

$$
H(k)=\lambda \sum_{\mu=x, y, z} \sigma_{\mu} \sin k_{\mu}+\tau_{y} \sigma_{y} M_{k},
$$

where $M_{k}=m+2-\cos k_{x}-\cos k_{z}, \tau_{\mu}$ are Pauli matrices acting in an orbital space, and $\sigma_{\mu}$ acts in a spin space. For this model we do not find situations in which closed Fermi loops form at the interface which are disconnected from the Weyl nodes. Nevertheless, three-dimensional closed orbits should still form in a magnetic field, which pass through the interface states. At small twist angles some of these loops will acquire an anomalously long period in the same way as was found above in the system with broken time-reversal symmetry. We again expect this to lead to magneto-oscillations of suppressed frequency.

In real materials, surfaces can have significantly larger numbers of Fermi arcs, which may connect among one another along arcs of nonvanishing curvature. At small twist angles we expect such systems will also host low-frequency magneto-oscillations. It is interesting to speculate that some such systems might also host closed Fermi loops as found in the two-band model, as well the elimination of open Fermi arcs connecting some of the bulk Weyl nodes. We leave these questions for future research. 


\section{ACKNOWLEDGMENTS}

This work was supported by the US-Israel Binational Science Foundation (Grant No. 2016130: G.M., H.A.F., E.S.; Grant No. 2018726: H.A.F., E.S.), by the NSF (Grants No. DMR-1506263, No. DMR-1506460, and No. DMR1914451), and by the Israel Science Foundation (ISF), Grant No. 231/14 (E.S.). The authors acknowledge the hospitality and support of the Aspen Center for Physics (Grant No. PHY1607611), where part of this work was done. G.M. is grateful to the Gordon and Betty Moore Foundation for sabbatical support at MIT, and the Lady Davis Foundation for sabbatical support at the Technion, while these ideas were being formed. H.A.F. acknowledges the support of the Research Corporation for Science Advancement through a Cottrell SEED Award. We also thank Sumathi Rao for illuminating conversations. All three of us thank the International Center for Theoretical Sciences, Bangalore, for its hospitality during a conference, when this work was being finalized.

\section{APPENDIX A: FERMI ARC STATES FOR A SINGLE SEMI-INFINITE SLAB: CONTINUUM APPROACH}

In this Appendix we discuss the continuum model, which has the advantage of being easy to generalize to the case of tunnel-coupled surfaces of Weyl semimetals twisted with respect to each other at arbitrary angle. The disadvantage is that one can only incorporate tunnel coupling between the surfaces perturbatively.

We start with our tight-binding Hamiltonian of Eq. (1) in the main text, with $t$ set equal to unity from the beginning (and $\left.t^{\prime}>0\right)$ :

$$
H=2 \sum_{\mu} f_{\mu} \sigma_{\mu}
$$

where $\quad f_{x}=2+\cos \left(k_{0}\right)-\cos \left(k_{x}\right)-\cos \left(k_{y}\right)-\cos \left(k_{z}\right) \equiv$ $1-\cos \left(k_{z}\right)+\tilde{f}_{x}, f_{y}=\sin \left(k_{y}\right)$, and $f_{z}=t^{\prime} \sin \left(k_{z}\right)$. (A special case of this model, $t^{\prime}=1$, was considered in Ref. [33].) We then employ a small $k_{z}$ expansion, yielding a continuum approximation in the $z$ direction (where the coordinate $z$ is measured in units of the lattice constant $a=1$ ). The functions $f_{x}, f_{z}$ now become

$$
\begin{aligned}
& f_{x}=\frac{1}{2} k_{z}^{2}+\tilde{f}_{x}, \quad \tilde{f}_{x}=1+\cos \left(k_{0}\right)-\cos \left(k_{x}\right)-\cos \left(k_{y}\right), \\
& f_{z}=t^{\prime} k_{z} .
\end{aligned}
$$

In a real-space representation of the $z$ direction, the Hamiltonian density acquires the form

$$
H\left(k_{x}, k_{y}, z\right)=-2 i t^{\prime} \sigma_{z} \frac{\partial}{\partial z}+\left(2 \tilde{f}_{x}-\frac{\partial^{2}}{\partial z^{2}}\right) \sigma_{x}+2 f_{y} \sigma_{y} .
$$

Considering a semi-infinite slab where $z \geqslant 0$, we then search for eigenvectors $\boldsymbol{\Phi}(z)$ of $H$ [with eigenvalues $E\left(k_{x}, k_{y}\right)$ ] which obey vanishing boundary conditions on the surface $z=0$ and decay in the bulk $z \rightarrow \infty$.

From Eq. (A3) it is apparent that $\boldsymbol{\Phi}(z)$ are linear combinations of eigenvectors of the form

$$
\boldsymbol{\Phi}_{\lambda}(z)=\boldsymbol{\Phi}_{\mathbf{0}} e^{-\lambda z},
$$

where $\operatorname{Re}\{\lambda\}>0$ and $\boldsymbol{\Phi}_{\mathbf{0}}$ is a (z-independent) two-component spinor. Substituting $\boldsymbol{\Phi}_{\lambda}$ in $H \boldsymbol{\Phi}_{\lambda}=E \boldsymbol{\Phi}_{\lambda}$, we obtain a quadratic equation for $\lambda$ as a function of $E, k_{x}, k_{y}$ with the solutions

$$
\lambda_{ \pm}^{2}=2\left[\tilde{f}_{x}+\left(t^{\prime}\right)^{2}\right] \pm \sqrt{4\left[\tilde{f}_{x}+\left(t^{\prime}\right)^{2}\right]^{2}+E^{2}-4\left[\tilde{f}_{x}^{2}+f_{y}^{2}\right]} .
$$

The corresponding eigenvectors are of the form Eq. (A4) with

$$
\boldsymbol{\Phi}_{\mathbf{0}}^{( \pm)} \equiv\left(\begin{array}{c}
u_{ \pm} \\
v_{ \pm}
\end{array}\right)=\left(\begin{array}{c}
2 \tilde{f}_{x}-2 i f_{y}-\lambda_{ \pm}^{2} \\
E-2 i t^{\prime} \lambda_{ \pm}
\end{array}\right) .
$$

The resulting eigenstates $\boldsymbol{\Phi}(z)=a_{+} \boldsymbol{\Phi}_{\lambda_{+}}(z)+a_{-} \boldsymbol{\Phi}_{\lambda_{-}}(z)$ obey the boundary condition $\boldsymbol{\Phi}(0)=0$ provided the coefficients $a_{ \pm}$ satisfy

$$
\begin{gathered}
M\left(\begin{array}{l}
a_{+} \\
a_{-}
\end{array}\right)=0 \text { with } \\
M=\left(\begin{array}{cc}
2 \tilde{f}_{x}-2 i f_{y}-\lambda_{+}^{2} & 2 \tilde{f}_{x}-2 i f_{y}-\lambda_{-}^{2} \\
E-2 i t^{\prime} \lambda_{+} & E-2 i t^{\prime} \lambda_{-}
\end{array}\right),
\end{gathered}
$$

which has a solution provided $\operatorname{det} M=0$. Assuming $\lambda_{+} \neq$ $\lambda_{-}$, this yields the equation

$$
-E\left(\lambda_{+}+\lambda_{-}\right)+4 i t^{\prime}\left(\tilde{f}_{x}-i f_{y}\right)+2 i t^{\prime} \lambda_{+} \lambda_{-} .
$$

Assuming that $\lambda_{+}, \lambda_{-}$are real, they must obey simultaneously

$$
-E\left(\lambda_{+}+\lambda_{-}\right)+4 t^{\prime} f_{y}=0 \text { and } 2 \tilde{f}_{x}=-\lambda_{+} \lambda_{-} .
$$

We next impose the requirement $\lambda_{+}, \lambda_{-}>0$, which implies that the second conditions in Eq. (A10) can be satisfied only provided $\tilde{f}_{x}<0$. Additionally, for the special case $E=0$ the first condition yields $f_{y}=0$. Recalling the definition of $\tilde{f}_{x}, f_{y}$ [Eq. (A2)], we conclude that a consistent solution for the surface states exists along the segment $k_{y}=0,\left|k_{x}\right|<k_{0}$. Notably, this is precisely the Fermi arc connecting the two Weyl nodes $\left( \pm k_{0}, 0\right)$. For arbitrary $E$, we combine Eqs. (A5) and (A10) to get

$$
\begin{aligned}
E & =2 f_{y}, \\
\lambda_{ \pm} & =t^{\prime} \pm \sqrt{\left(t^{\prime}\right)^{2}+2 \tilde{f}_{x}} .
\end{aligned}
$$

Interestingly, when substituted in Eq. (A6) to obtain the eigenvectors, we find

$$
\left(\begin{array}{l}
u_{+} \\
v_{+}
\end{array}\right)=\left(\begin{array}{l}
u_{-} \\
v_{-}
\end{array}\right)=\frac{1}{\sqrt{2}}\left(\begin{array}{l}
1 \\
i
\end{array}\right)
$$

i.e., the surface states are spin polarized along the $\sigma_{y}$ direction, as stated in the main text.

\section{APPENDIX B: FERMI ARC STATES FOR A SINGLE SEMI-INFINITE SLAB: LATTICE CALCULATION}

In this Appendix we develop a formulation for the case of a single semi-infinite slab. We consider the lattice Hamiltonian Eq. (A1) and transform to real space in the $z$ direction, with the lattice sites in this direction labeled by $n$. The momenta $k_{x}, k_{y}$ will be left as parameters. We define the lattice Fourier transform by

$$
\begin{aligned}
& c_{n}\left(k_{x}, k_{y}\right)=\frac{1}{\sqrt{N_{z}}} \sum_{k_{z}} e^{i k_{z} n a} c\left(k_{x}, k_{y}, k_{z}\right), \\
& c\left(k_{x}, k_{y}, k_{z}\right)=\frac{1}{\sqrt{N_{z}}} \sum_{n} e^{-i k_{z} n a} c_{n}\left(k_{x}, k_{y}\right),
\end{aligned}
$$


where the lattice spacing is $a \equiv 1$ and we have suppressed the spin index in the fermion destruction operators $c\left(k_{x}, k_{y}, k_{z}\right)$, $c_{n}\left(k_{x}, k_{y}\right)$. After a bit of algebra we obtain

$$
\begin{aligned}
H\left(k_{x}, k_{y}\right)= & \sum_{n}\left\{-c_{n+1}^{\dagger}\left(\sigma_{x}-i t^{\prime} \sigma_{z}\right) c_{n}-c_{n}^{\dagger}\left(\sigma_{x}+i t^{\prime} \sigma_{z}\right) c_{n+1}\right. \\
& \left.+2 c_{n}^{\dagger}\left[\left(1+\tilde{f}_{x}\right) \sigma_{x}+f_{y} \sigma_{y}\right] c_{n}\right\}
\end{aligned}
$$

In the above we have suppressed the $k_{x}, k_{y}$ arguments in all fermion operators for notational simplicity. For further analysis, it is convenient to rotate the $\sigma$ matrices by $\frac{\pi}{2}$ around the $x$ axis, which has the virtue of making the Hamiltonian purely real. In this basis, the Hamiltonian is

$$
\begin{aligned}
H\left(k_{x}, k_{y}\right)= & \sum_{n}\left\{-c_{n+1}^{\dagger}\left(\sigma_{x}+i t^{\prime} \sigma_{y}\right) c_{n}-c_{n}^{\dagger}\left(\sigma_{x}-i t^{\prime} \sigma_{y}\right) c_{n+1}\right. \\
& \left.+2 c_{n}^{\dagger}\left[\left(1+\tilde{f}_{x}\right) \sigma_{x}+f_{y} \sigma_{z}\right] c_{n}\right\}
\end{aligned}
$$

Since the Hamiltonian is real, the wave function can also be chosen real. This will play an important role in the counting argument that shows that the conditions to have a surface state can be met.

To consider a semi-infinite slab, we simply restrict the sum over $n$ to either non-negative or non-positive integers. For specificity, let us consider the bottom surface of a semi-infinite slab, with $n=0,1,2, \ldots$ We want solutions that decay into the bulk, so we make the eigenstate ansatz

$$
\left|E, k_{x}, k_{y}\right\rangle=\sum_{n=0}^{\infty} \sum_{s=\uparrow, \downarrow} u^{n} \Phi_{0 s}|n, s\rangle,
$$

where $|u|<1$ for the state to be normalizable. Demanding $H\left|E, k_{x}, k_{y}\right\rangle=E\left|E, k_{x}, k_{y}\right\rangle$ leads to the matrix condition on $\Phi_{0 s}$, for $n>0$,

$$
\left\{u^{2}\left(\sigma_{x}-i t^{\prime} \sigma_{y}\right)+u\left[E-2\left(1+\tilde{f}_{x}\right) \sigma_{x}-2 \sin \left(k_{y}\right) \sigma_{z}\right]+\sigma_{x}+i t^{\prime} \sigma_{y}\right\} \Phi_{0} \equiv M \Phi_{0}=0 .
$$

In explicit terms, the matrix $M$ is

$$
M=\left(\begin{array}{cc}
u\left(E-2 f_{y}\right) & 1+u^{2}-2\left(1+\tilde{f}_{x}\right)+t^{\prime}\left(1-u^{2}\right) \\
1+u^{2}-2 u\left(1+\tilde{f}_{x}\right)-t^{\prime}\left(1-u^{2}\right) & u\left(E+2 f_{y}\right)
\end{array}\right) .
$$

Since the matrix $M$ has a zero eigenvector, its determinant should vanish. This gives a quartic equation for $u$, which, by changing to the variable

$$
\xi=u+\frac{1}{u},
$$

can be recast as a quadratic equation,

$$
\begin{aligned}
& \xi^{2}\left(1-t^{\prime 2}\right)-4 \xi\left(1+\tilde{f}_{x}\right)-\left[E^{2}-4\left(1+\tilde{f}_{x}\right)^{2}-4 f_{y}^{2}-4 t^{\prime 2}\right] \\
& \quad=0 .
\end{aligned}
$$

Clearly, we can solve this quadratic and then the auxiliary quadratic $u^{2}-u \xi+1=0$ to obtain four values of $u$. The structure of this auxiliary quadratic shows that the values of $u$ occur in pairs whose product is unity. Thus, either both roots are real, with one being less than unity and the other greater, or they are unimodular and complex conjugates of each other. In the latter case the wave functions will not be normalizable, and thus there will be no surface modes.

It is interesting to consider the relation of our approach to that of Ref. [33], which is restricted to $t^{\prime}=1$. Clearly, $t^{\prime}=1$ is singular in the sense that the quadratic equation Eq. (B9) becomes a linear one. This means one of the roots has been pushed off to infinity. Solving the auxiliary quadratic, we see that there are only two physically acceptable roots for $u$ in this case, with only one of them being less than unity in magnitude. Reference [33] presents many elegant results in this simplified model, but one might worry that the results of the fine-tuned $t^{\prime}=1$ model may not be generic. Our analysis demonstrates that in fact they are.

Going back to our problem, let us assume that we have obtained two real values of $u$, say $u_{1}, u_{2}$ such that $\left|u_{1}\right|<$ $1,\left|u_{2}\right|<1$. The next step is to find the eigenvectors corresponding to these values of $u_{i}$ by solving $M\left(u_{i}\right) \Phi_{0}^{i}=0$. The final step is to find a linear combination of these solutions

$$
\Phi(n)=\alpha_{1} u_{1}^{n} \Phi_{0}^{1}+\alpha_{2} u_{2}^{n} \Phi_{0}^{2}
$$

that satisfies the boundary condition on the surface, which can be written as

$$
\Phi(n=-1)=0 .
$$

Since the $u_{i}$ as well as the wave functions are real, so are $\alpha_{i}$. The normalization is immaterial for satisfying Eq. (B11), which means we can set $\alpha_{1}=1$. Thus, for a given $k_{x}, k_{y}$, we have one real free parameter $\alpha_{2}$ and another real free parameter $E$. With these two parameters we can indeed satisfy the two conditions represented by Eq. (B11). This means a solution exists as long as we can find two real $u(b)_{i}$, with $\left|u_{i}^{(b)}\right|<1$. [Note that the superscript $(b)$ denotes the bottom surface.]

Given a $u$ which solves the characteristic equation, we can find the eigenvector corresponding to it by solving Eq. (B6). For future reference, the un-normalized spinor can be expressed in two equivalent ways,

$$
\begin{gathered}
\Phi_{0}\left(u, E, k_{x}, k_{y}\right)=\left(\begin{array}{c}
1 \\
-\frac{u\left(E-2 f_{y}\right)}{u^{2}\left(1+t^{\prime}\right)-2 u F_{x}+1-t^{\prime}}
\end{array}\right) \\
\text { or } \left.\begin{array}{c}
-\frac{u\left(E+2 f_{y}\right)}{u^{2}\left(1-t^{\prime}\right)-2 u F_{x}+1+t^{\prime}} \\
1
\end{array}\right) .
\end{gathered}
$$

The solutions turn out to be particularly simple if one assumes $0<t^{\prime}=\sin \phi<1$ and $k_{0}<\phi$. For the bottom surface of a semi-infinite slab, the energy is

$$
E^{(b)}\left(k_{x}, k_{y}\right)=2 f_{y}=2 \sin k_{y} .
$$




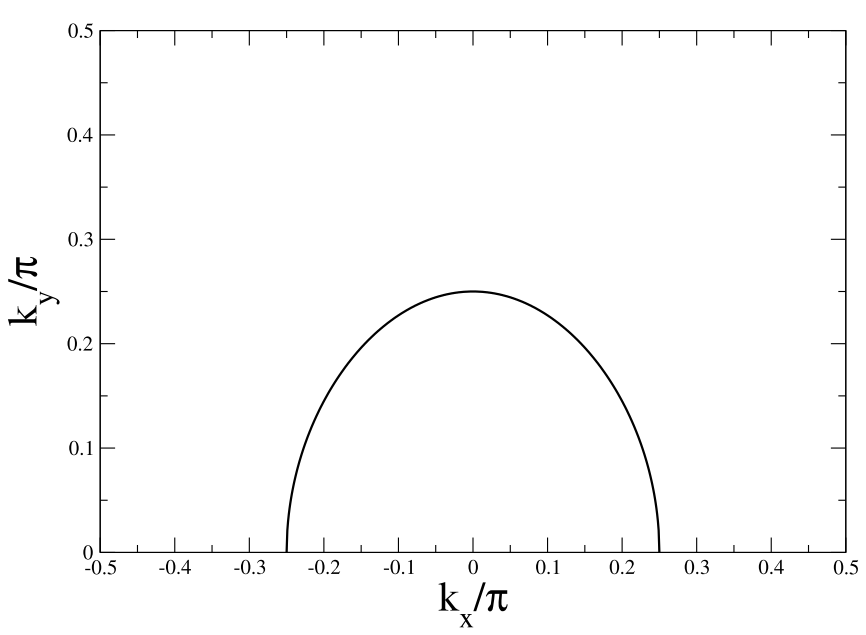

FIG. 3. The region of the surface BZ where Fermi arcs with $E>$ 0 on the bottom surface of a semi-infinite slab are allowed in our model. The parameters we have chosen for this plot are $k_{0}=\frac{\pi}{4}$ and $t^{\prime}=0.8$. There is an identical region for $k_{y}<0$ which corresponds to arcs with $E<0$, again for the bottom surface of a semi-infinite slab, which is not shown.

(Note this same result was obtained previously [33] for the specific case $t^{\prime}=1$.) Defining $F_{x}=1+\tilde{f}_{x}=\cos k_{0}+$ $\frac{1}{2}\left(\sin ^{2} \frac{k_{x}}{2}+\sin ^{2} \frac{k_{y}}{2}\right)$, we find that the two $u_{i}$ we want are solutions to the vanishing of the lower left entry of the matrix $M$. Thus

$$
\begin{aligned}
1 & +u^{2}-2 F_{x} u-t^{\prime}\left(1-u^{2}\right) \\
& =u^{2}(1+\sin \phi)-2 F_{x} u+(1-\sin \phi)=0,
\end{aligned}
$$

yielding the solutions

$$
u_{ \pm}^{(b)}=\frac{F_{x} \pm \sqrt{F_{x}^{2}-\cos ^{2} \phi}}{1+\sin \phi} .
$$

The Fermi arcs persist within a region of the surface BZ where $F_{x}<1$, or equivalently

$$
\cos k_{x}+\cos k_{y}>1+\cos k_{0} .
$$

The region is illustrated in Fig. 3 for $k_{0}=\frac{\pi}{4}$. In our simple model the spinors for the bottom surface are always

$$
\Phi_{i}^{(b)}=\left(\begin{array}{l}
1 \\
0
\end{array}\right) .
$$

The eigenstates are spin polarized along the $\sigma_{z}$ direction. Recalling the rotation of the Pauli matrices implemented in the beginning of this analysis, this corresponds to polarization along the $\sigma_{y}$ direction in the original model Eq. (1).

Now consider the top surface of a semi-infinite slab. In the lattice Hamiltonian the values of $n$ now lie in the range $n=$ $0,-1,-2,-3, \ldots,-\infty$. Instead of the ansatz we made for the bottom surface, Eq. (B5), we now make the ansatz

$$
\left|E, k_{x}, k_{y}\right\rangle=\sum_{n=0}^{\infty} \sum_{s=\uparrow, \downarrow} u^{-n} \Phi_{0 s}|n, s\rangle .
$$

Since $n$ now goes to large negative values we see that we again want values of the two $u_{i}$ which satisfy $\left|u_{i}\right|<1$.

The condition for the bulk to satisfy the eigenvalue equation remains the same as Eq. (B6), and the four roots of the characteristic equation remain the same. Since, in our model, we have shown that each root occurs with its reciprocal, we simply choose, in the appropriate region [Eq. (B14)], the two values of $u$ to be

$$
u_{i}^{(t)}=u_{i}^{(b)} .
$$

For the bottom surface the energy is

$$
E^{(t)}\left(k_{x}, k_{y}\right)=-2 f_{y}=-2 \sin k_{y}
$$

and the spinor is

$$
\Phi^{(t)}=\left(\begin{array}{l}
0 \\
1
\end{array}\right) .
$$

At $E=0$ the Fermi arcs join the projections of the Weyl points onto the surface BZ. The Fermi arc state on the bottom surface has a (group) velocity in the positive $y$ direction while that on the top surface has a group velocity in the negative $y$ direction.

We finally comment on the comparison with the continuum approximation described in Sec. I. Within the lattice approach, the eigenstates are parametrized by the multiplicative factor $u$ which denotes a decay of the amplitude within a single lattice constant along the $z$ direction. The continuum solution Eq. (A4) for $z=a=1$ implies that, in the appropriate limit, one should recover $u=e^{-\lambda}$. In particular, the continuum approximation is valid for $\lambda_{ \pm} \ll 1$; from the expression Eq. (A11), this is obeyed for $t^{\prime} \ll 1$. For simplicity, we further consider a range of $k_{x}$ where $\tilde{f}_{x} \ll t^{\prime} \ll 1$ (so that $\lambda_{ \pm}$are real). We then recall Eq. (B13) for $u_{ \pm}^{(b)}$ (with $t^{\prime}=\sin \phi$ and $\tilde{f}_{x}=F_{x}-1$ ), and expand $-\ln \left\{u_{ \pm}^{(b)}\right\}$ to leading order in $t^{\prime}, \tilde{f}_{x}$, to find

$$
\begin{gathered}
-\ln \left\{u_{ \pm}^{(b)}\right\}=-\ln \left\{F_{x} \pm \sqrt{F_{x}^{2}-1+\left(t^{\prime}\right)^{2}}\right\}+ \\
\ln \left\{1+t^{\prime}\right\} \approx t^{\prime}-\tilde{f}_{x} \mp \sqrt{2 \tilde{f}_{x}+\left(t^{\prime}\right)^{2}} \approx \lambda_{\mp} .
\end{gathered}
$$

Noting that, additionally, the solutions for the energy $E$ and eigenvectors are found to be identical, the continuum limit is indeed recovered in the appropriate limit.

\section{APPENDIX C: TWO SLABS ROTATED BY $\frac{\pi}{2}$}

We next consider the case of two slabs of identical Weyl semimetal twisted with respect to each other by $\frac{\pi}{2}$ and tunnel coupled to one another. The advantage of the lattice formulation is that for twist angles that are multiples of $\pi / 2$, it allows a treatment that is nonperturbative in the tunnel coupling.

The key premise of the main text is that when the top surface of one slab of WSM is tunnel coupled to the bottom surface of another WSM, the Fermi arcs on the two surfaces will hybridize and reconstruct. Generically, since at arbitrary angles of rotation of one surface with respect to the other there is no true periodicity and thus no surface BZ, this problem cannot be handled by strictly lattice methods. However, one simple case that can be handled by lattice methods is when two slabs of the same WSM (assumed to have a square lattice in the $x y$ plane) are rotated with respect to each other by $\frac{\pi}{2}$ and tunnel coupled. In the following we will assume that the tunnel coupling between the two surfaces in the $z$ direction is identical in form to the coupling in the bulk, but may differ 
in magnitude. We will use $c_{n}, n=0,1,2, \ldots, \infty$, for the operators of the top slab, and $d_{n}, n=0,-1,-2, \ldots,-\infty$, for the operators of the bottom slab, suppressing the spin and $k_{x}, k_{y}$ labels. Bearing in mind that we are rotating the top slab by $\frac{\pi}{2}$ the total Hamiltonian is

$$
\begin{aligned}
H= & \sum_{n=0}^{-\infty}\left\{d_{n}^{\dagger}\left[2 \sin k_{y} \sigma_{z}+2 \sigma_{x}\left(2+\cos k_{0}-\cos k_{x}-\cos k_{y}\right)\right] d_{n}\right. \\
& \left.-d_{n}^{\dagger}\left(\sigma_{x}-i t^{\prime} \sigma_{y}\right) d_{n-1}-d_{n-1}^{\dagger}\left(\sigma_{x}+i t^{\prime} \sigma_{y}\right) d_{n}\right\} \\
& +\sum_{n=0}^{\infty}\left\{c _ { n } ^ { \dagger } \left[-2 \sin k_{x} \sigma_{z}+2 \sigma_{x}\left(2+\cos k_{0}-\cos k_{x}\right.\right.\right. \\
& \left.\left.\left.-\cos k_{y}\right)\right] c_{n}-c_{n+1}^{\dagger}\left(\sigma_{x}-i t^{\prime} \sigma_{y}\right) c_{n}-c_{n}^{\dagger}\left(\sigma_{x}+i t^{\prime} \sigma_{y}\right) c_{n+1}\right\} \\
& -\kappa d_{0}^{\dagger}\left(\sigma_{x}+i t^{\prime} \sigma_{y}\right) c_{0}-\kappa c_{0}^{\dagger}\left(\sigma_{x}-i t^{\prime} \sigma_{y}\right) d_{0} .
\end{aligned}
$$

Here $\kappa$ parameterizes the strength of the tunnel coupling between the two surfaces. Note the appearance of $-2 \sin k_{x}$ as the coefficient of $\sigma_{z}$ in the site-diagonal term in the Hamiltonian for the top slab. This is because the slab has been rotated by $\frac{\pi}{2}$, leading to $k_{x} \rightarrow k_{y}, k_{y} \rightarrow-k_{x}$. To reduce notational complexity in what follows, we introduce the following definitions:

$$
\begin{aligned}
& f_{y}^{(t)}\left(k_{x}, k_{y}\right)=-\sin k_{x}, \\
& f_{y}^{(b)}\left(k_{x}, k_{y}\right)=\sin k_{y},
\end{aligned}
$$

$$
\left(\begin{array}{ccc}
\left(u_{1}^{(t)}\right)^{-1} & \left(u_{2}^{(t)}\right)^{-1} & -\kappa g_{1}^{(b)} \\
g_{1}^{(t)}\left(u_{1}^{(t)}\right)^{-1} & g_{2}^{(t)}\left(u_{2}^{(t)}\right)^{-1} & -\kappa \\
\kappa & \kappa & -g_{1}^{(b)}\left(u_{1}^{(b)}\right)^{-1} \\
\kappa g_{1}^{(t)} & \kappa g_{2}^{(t)} & -\left(u_{1}^{(b)}\right)^{-1}
\end{array}\right.
$$

Now suppose we want to find the reconstructed Fermi arc at the interface for a given value of $E$. For a solution to exist the determinant of the matrix $M$ must vanish. The only free variables left in $M$ once $E$ is fixed are $k_{x}, k_{y}$, and $\operatorname{det}(M)=0$ puts one condition on them. This is the implicit equation for the reconstructed Fermi arc. In contrast to the results for the semi-infinite slabs, the reconstructed Fermi arcs for $\kappa \neq 0$ do depend on the value of $t^{\prime}$.

In Fig. 4 we show the reconstructed Fermi arc for $E=0$ for different values of the interslab coupling $\kappa$. Only the first quadrant of the surface $\mathrm{BZ}$ is shown, with a similar reconstruction occurring in the third quadrant.

\section{APPENDIX D: CURVED FERMI ARCS AND DETACHMENT FROM WEYL POINTS}

In this final Appendix we modify the Hamiltonian of Eq. (A1) to obtain curved Fermi arcs, and show by a lattice calculation that when the tunnel coupling is sufficiently strong, the Fermi arcs can detach from the projections of the Weyl points, and form closed loops in the surface BZ.

In the main text we noted that at the arcless angles, when the projections of the Weyl points of positive chirality coincide in the surface Brillouin zone (and likewise for the

$$
\begin{aligned}
F_{x}^{(t)} & =F_{x}^{(b)} \equiv F_{x}=2+\cos k_{0}-\cos k_{x}-\cos k_{y}, \\
g^{(t)}(u) & =-\frac{u\left(E-f_{y}^{(t)}\right)}{u^{2}\left(1+t^{\prime}\right)-2 u F_{x}+1-t^{\prime}}, \\
g^{(b)}(u) & =-\frac{u\left(E-f_{y}^{(b)}\right)}{u^{2}\left(1-t^{\prime}\right)-2 u F_{x}+1+t^{\prime}} .
\end{aligned}
$$

For a given $E, k_{x}, k_{y}$ let us call the two appropriate roots in the top slab $u_{i}^{(t)}$, and the two in the bottom slab $u_{i}^{(b)}$. Generically these will not be the same because $f_{y}^{(t)}$ and $f_{y}^{(b)}$ differ. Further defining

$$
g_{i}^{(t)} \equiv g^{(t)}\left(u_{i}^{(t)}\right), \quad g_{i}^{(b)} \equiv g^{(b)}\left(u_{i}^{(b)}\right),
$$

we write the wave functions in the top and bottom slabs as

$$
\begin{gathered}
\Phi^{(t)}(n)=\alpha_{1}\left(u_{1}^{(t)}\right)^{n}\left(\begin{array}{c}
1 \\
g_{1}^{(t)}
\end{array}\right)+\alpha_{2}\left(u_{2}^{(t)}\right)^{n}\left(\begin{array}{c}
1 \\
g_{2}^{(t)}
\end{array}\right), \\
\Phi^{(b)}(n)=\beta_{1}\left(u_{1}^{(b)}\right)^{-n}\left(\begin{array}{c}
g_{1}^{(b)} \\
1
\end{array}\right)+\beta_{2}\left(u_{2}^{(b)}\right)^{-n}\left(\begin{array}{c}
g_{2}^{(t)} \\
1
\end{array}\right) .
\end{gathered}
$$

The boundary conditions at the interface can be compactly written as

$$
\Phi^{(t)}(n=-1)=\kappa \Phi^{(b)}(0), \quad \Phi^{(b)}(n=+1)=\kappa \Phi^{(t)}(0) .
$$

In explicit form, these four equations can be written as

$$
\left.\begin{array}{c}
-\kappa g_{2}^{(b)} \\
-\kappa \\
-g_{2}^{(b)}\left(u_{2}^{(b)}\right)^{-1} \\
-\left(u_{2}^{(b)}\right)^{-1}
\end{array}\right)\left(\begin{array}{l}
\alpha_{1} \\
\alpha_{2} \\
\beta_{1} \\
\beta_{2}
\end{array}\right) \equiv M\left(\begin{array}{c}
\alpha_{1} \\
\alpha_{2} \\
\beta_{1} \\
\beta_{2}
\end{array}\right)=0
$$

negative chirality Weyl points), the Fermi arcs may form closed loops which are detached from the Weyl points. To demonstrate this requires a nonperturbative calculation in the coupling between the two surfaces. Analogous behavior has been demonstrated for $t^{\prime}=1$ [33], but the special properties of that parameter choice (discussed above) leave open the question of whether such behavior is generic. To address this, we present in this section an analogous calculation demonstrating that Fermi loops can indeed detach from the projections of the Weyl points for sufficiently strong tunnel coupling, without fine-tuning of parameters.

We modify our model slightly to produce curved Fermi arcs, by changing the functional form of $f_{y}$ to

$$
f_{y}\left(k_{x}, k_{y} ; \lambda\right)=\sin k_{y}+\lambda\left(\cos k_{x}-\cos k_{0}\right) .
$$

It is easy to see that the bulk Weyl points remain unchanged, regardless of the value of $\lambda$. All the manipulations of Appendix B go through as before. From Eq. (B12), for the bottom surface of a semi-infinite slab, the Fermi arc at $E=0$ is given by

$$
E=2 f_{y}\left(k_{x}, k_{y} ; \lambda\right)=0 .
$$




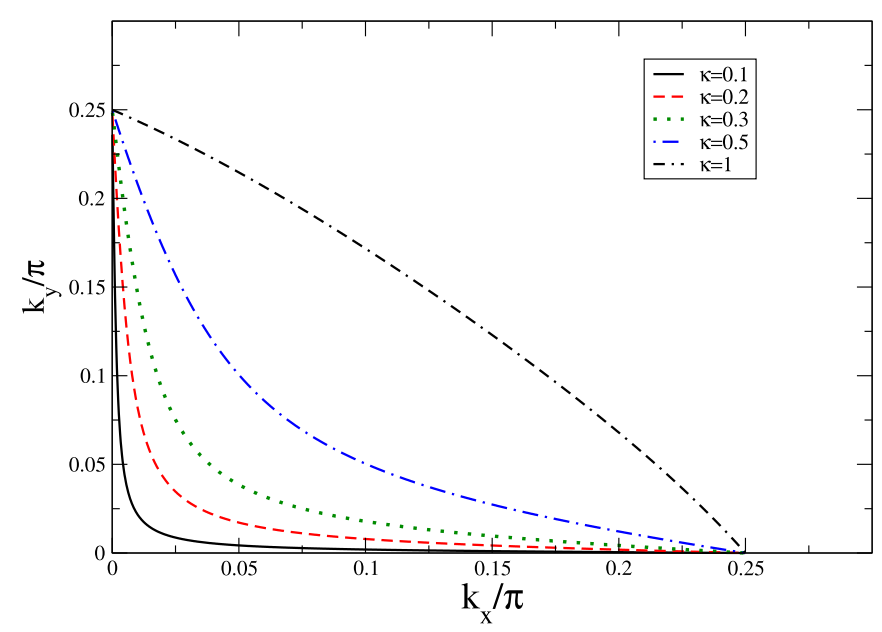

FIG. 4. Reconstructed Fermi arcs for two semi-infinite slabs of identical Weyl semimetals rotated by $\frac{\pi}{2}$ with respect to each other and the free surfaces tunnel coupled with a strength $\kappa$. The parameters we have chosen for this figure are $k_{0}=\frac{\pi}{4}$ and $t^{\prime}=\sin \frac{\pi}{3}$. Only the first quadrant is shown. There is an identical reconstructed Fermi arc in the third quadrant as well. At zero tunnel coupling the Fermi arcs are unreconstructed. The Fermi arc belonging to the top slab will be the vertical line $k_{x}=0,-k_{0}<k_{y}<k_{0}$, while the Fermi arc belonging to the bottom slab will be the horizontal line $-k_{0}<$ $k_{x}<k_{0}, k_{y}=0$. The projections of the Weyl points with positive monopole number are at $\left(0, k_{0}\right)$ (top slab) and $\left(k_{0}, 0\right)$ (bottom slab). As $\kappa$ increases, the surface states hybridize, and the Fermi arcs reconstruct to reconnect the projections of the + monopoles of the two slabs together and the - monopoles together.

Let us choose the top semi-infinite slab as above with $\lambda_{t}>0$, while the bottom slab has the same form with $\lambda_{b}=-\lambda_{t}$. The two lattices are in registry with each other, and the surfaces are brought into proximity, with the coupling being of the same form as in Eq. (C1). The formalism of Appendix C is

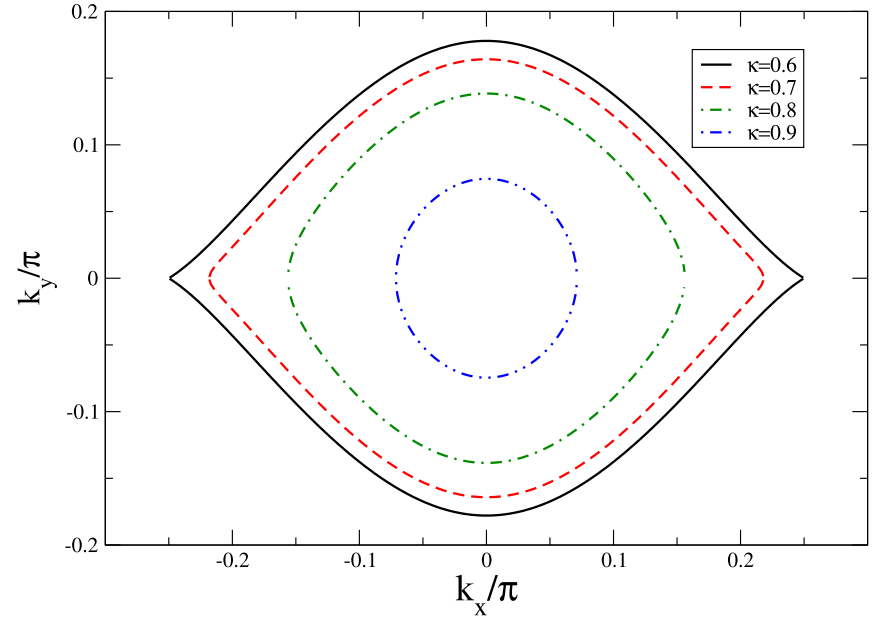

FIG. 5. Evolution of the Fermi arcs of the interface of two slabs with a tunnel coupling $\kappa$ at the surface. Both slabs have bulk Weyl points at $\left( \pm k_{0}, 0,0\right)$. The values of the parameters are $k_{0}=\frac{\pi}{4}, t^{\prime}=$ $\sin \frac{\pi}{3}$. The top slab has $\lambda=2$ [see Eq. (D1)], while the bottom slab has $\lambda=-2$. For small values of the tunnel coupling $\kappa$, the Fermi arcs end at the projections of the Weyl points on the surface BZ. However, beyond a critical $\kappa^{*} \approx 0.65$ the Fermi arcs detach from the projections of the Weyl points and form an independent closed loop in the surface Brillouin zone. This loop shrinks as $\kappa$ increases, and vanishes for $\kappa>1$.

general enough to accommodate this situation with the appropriate replacements for $f_{y}^{(t)}, f_{y}^{(b)}$, so the implicit equation for the Fermi arcs is once again given by the vanishing of the determinant of the appropriate $M$ matrix.

In Fig. 5 we show the evolution of the Fermi arcs as the coupling strength $\kappa$ between the two surfaces is increased. There is a critical value of $\kappa^{*} \approx 0.65$ beyond which the Fermi arcs detach from the projections of the Weyl points and form a closed loop detached from the Weyl point projections.
[1] S. Jia, S.-Y. Xu, and M. Z. Hasan, Nat. Mater. 15, 1140 (2016).

[2] N. P. Armitage, E. J. Mele, and A. Vishwanath, Rev. Mod. Phys. 90, 015001 (2018).

[3] C.-L. Zhang, S.-Y. Xu, I. Belopolski, Z. Yuan, Z. Lin, B. Tong, G. Bian, N. Alidoust, C.-C. Lee, S.-M. Huang et al., Nat. Commun. 7, 10735 (2016).

[4] S. Murakami, New J. Phys. 9, 356 (2007).

[5] X. Wan, A. M. Turner, A. Vishwanath, and S. Y. Savrasov, Phys. Rev. B 83, 205101 (2011).

[6] K.-Y. Yang, Y.-M. Lu, and Y. Ran, Phys. Rev. B 84, 075129 (2011).

[7] G. Xu, H. Weng, Z. Wang, X. Dai, and Z. Fang, Phys. Rev. Lett. 107, 186806 (2011).

[8] A. A. Burkov and L. Balents, Phys. Rev. Lett. 107, 127205 (2011).

[9] B. Q. Lv, H. M. Weng, B. B. Fu, X. P. Wang, H. Miao, J. Ma, P. Richard, X. C. Huang, L. X. Zhao, G. F. Chen et al., Phys. Rev. X 5, 031013 (2015).

[10] S.-Y. Xu, I. Belopolski, N. Alidoust, M. Neupane, G. Bian, C. Zhang, R. Sankar, G. Chang, Z. Yuan, C.-C. Lee et al., Science 349, 613 (2015).
[11] S.-Y. Xu, N. Alidoust, I. Belopolski, Z. Yuan, G. Bian, T.-R. Chang, H. Zheng, V. N. Strocov, D. S. Sanchez, G. Chang et al., Nat. Phys. 11, 748 (2015).

[12] H. Inoue, A. Gyenis, Z. Wang, J. Li, S. W. Oh, S. Jiang, N. Ni, B. A. Bernevig, and A. Yazdani, Science 351, 1184 (2016).

[13] E. H. da Silva Neto, Science 365, 1248 (2019).

[14] I. Belopolski, K. Manna, D. S. Sanchez, G. Chang, B. Ernst, J. Yin, S. S. Zhang, T. Cochran, N. Shumiya, H. Zheng et al., Science 365, 1278 (2019).

[15] D. F. Liu, A. J. Liang, E. K. Liu, Q. N. Xu, Y. W. Li, C. Chen, D. Pei, W. J. Shi, S. K. Mo, P. Dudin et al., Science 365, 1282 (2019).

[16] N. Morali, R. Batabyal, P. K. Nag, E. Liu, Q. Xu, Y. Sun, B. Yan, C. Felser, N. Avraham, and H. Beidenkopf, Science 365, 1286 (2019).

[17] T. Jungwirth, Q. Niu, and A. H. MacDonald, Phys. Rev. Lett. 88, 207208 (2002)

[18] Z. Fang, N. Nagaosa, K. S. Takahashi, A. Asamitsu, R. Mathieu, T. Ogasawara, H. Yamada, M. Kawasaki, Y. Tokura, and K. Terakura, Science 302, 92 (2003). 
[19] S. A. Parameswaran, T. Grover, D. A. Abanin, D. A. Pesin, and A. Vishwanath, Phys. Rev. X 4, 031035 (2014).

[20] Y. Baum, E. Berg, S. A. Parameswaran, and A. Stern, Phys. Rev. X 5, 041046 (2015).

[21] C. Zhang, E. Zhang, W. Wang, Y. Liu, Z.-G. Chen, S. Lu, S. Liang, J. Cao, X. Yuan, L. Tang et al., Nat. Commun. 8, 13741 (2017).

[22] T. Ojanen, Phys. Rev. B 87, 245112 (2013).

[23] A. C. Potter, I. Kimchi, and A. Vishwanath, Nat. Commun. 5, 5161 (2014).

[24] P. J. W. Moll, N. L. Nair, T. Helm, A. C. Potter, I. Kimchi, A. Vishwanath, and J. G. Analytis, Nature (London) 535, 266 (2016).

[25] C. Zhang, Y. Zhang, X. Yuan, S. Lu, J. Zhang, A. Narayan, Y. Liu, H. Zhang, Z. Ni, R. Liu et al., Nature (London) 565, 331 (2019).

[26] C. M. Wang, H.-P. Sun, H.-Z. Lu, and X. C. Xie, Phys. Rev. Lett. 119, 136806 (2017).

[27] R. Bistritzer and A. H. MacDonald, Proc. Natl. Acad. Sci. USA 108, 12233 (2011).

[28] P. San-Jose, J. González, and F. Guinea, Phys. Rev. Lett. 108, 216802 (2012).

[29] K. S. Novoselov, A. Mishchenko, A. Carvalho, and A. H. Castro Neto, Science 353, aac9439 (2016).

[30] D. L. Duong, S. J. Yun, and Y. H. Lee, ACS Nano 11, 11803 (2017).
[31] Y. Cao, V. Fatemi, A. Demir, S. Fang, S. L. Tomarken, J. Y. Luo, J. D. Sanchez-Yamagishi, K. Watanabe, T. Taniguchi, E. Kaxiras et al., Nature (London) 556, 80 (2018).

[32] Y. Cao, V. Fatemi, S. Fang, K. Watanabe, T. Taniguchi, E. Kaxiras, and P. Jarillo-Herrero, Nature (London) 556, 43 (2018).

[33] V. Dwivedi, Phys. Rev. B 97, 064201 (2018).

[34] H. Ishida and A. Liebsch, Phys. Rev. B 98, 195426 (2018).

[35] A. Ardavan, J. M. Schrama, S. J. Blundell, J. Singleton, W. Hayes, M. Kurmoo, P. Day, and P. Goy, Phys. Rev. Lett. 81, 713 (1998).

[36] C.-X. Liu, X.-L. Qi, H. J. Zhang, X. Dai, Z. Fang, and S.-C. Zhang, Phys. Rev. B 82, 045122 (2010).

[37] P. G. Silvestrov, P. W. Brouwer, and E. G. Mishchenko, Phys. Rev. B 86, 075302 (2012).

[38] L. Brey and H. A. Fertig, Phys. Rev. B 89, 085305 (2014).

[39] Note that these equations do not contain Berry's curvature terms, as the Fermi arc states are spin polarized, and the phases of $w_{d, u}$ are not expected to have significant $\mathbf{k}$ dependence in the regions where Fermi arcs on opposite sides of the interface are degenerate.

[40] C.-K. Lu and H. A. Fertig, Phys. Rev. B 90, 115436 (2014).

[41] M. M. Vazifeh and M. Franz, Phys. Rev. Lett. 111, 027201 (2013).

[42] S. Verma, D. Giri, H. A. Fertig, and A. Kundu, Phys. Rev. B 101, 085419 (2020). 